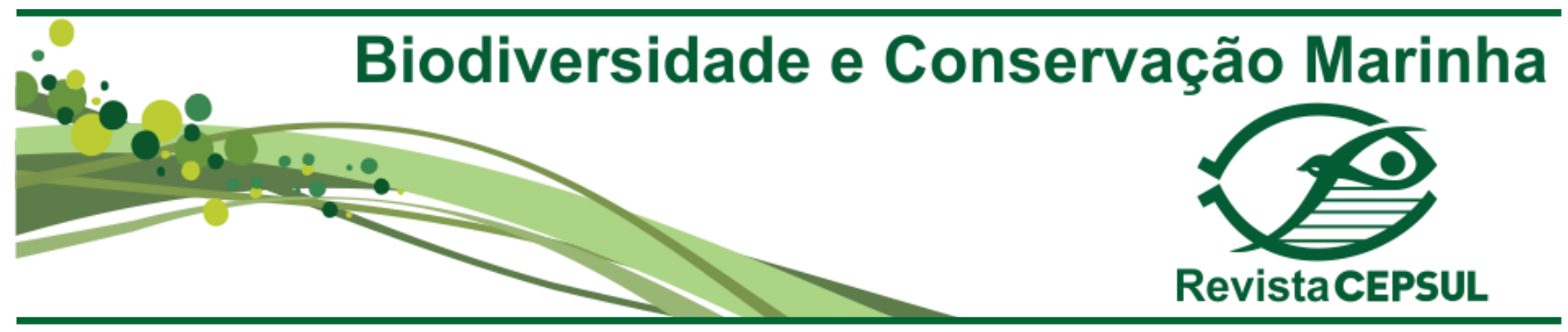

ARTIGO

\title{
Biologia e pesca do camarão sete-barbas \\ (Xiphopenaeus kroyeri, Heller 1862) no Balneário Barra do Sul, SC
}

\author{
LUIZ FERNANDO RODRIGUES ${ }^{1}$, HARRY BOOS ${ }^{1} \&$ JOAQUIM OLINTO BRANCO ${ }^{2}$
}

\begin{abstract}
${ }^{1}$ Centro Nacional de Pesquisa e Conservação da Biodiversidade Marinha do Sudeste e Sul - CEPSUL/ICMBio, Av. Ministro Victor Konder, 374, CEP - 88301-70o, Itajaí, SC, Brasil, harry.boos-junior@icmbio.gov.br;

${ }^{2}$ Centro de Ciências Tecnológicas da Terra e do Mar , Universidade do Vale do Itajaí - CTTMar/UNIVALI, Rua Uruguai, 458 ( Lab. de Biologia), CEP - 88302-202, Itajaí, SC, Brasil.
\end{abstract}

\begin{abstract}
Resumo. A produção desembarcada do camarão sete-barbas no Brasil mostrou uma redução considerável nos últimos anos quando comparada a produção anteriormente descrita. Na avaliação do risco de extinção, a espécie foi categorizada com “Dados Insuficientes - DD”, neste sentido, este trabalho preenche algumas das lacunas no conhecimento do camarão sete-barbas em uma tradicional área de pesca do Estado de Santa Catarina. Foram realizadas, entre fevereiro de 1993 a novembro de 1994, 41 amostragens sendo observados 14.873 indivíduos. As fêmeas predominaram na maioria dos meses, com diferença significativa na proporção de machos e fêmeas. Nos machos o comprimento do cefalotórax variou entre 6 e $28 \mathrm{~mm}$. Já as fêmeas, independente do estádio de maturação gonadal, apresentaram comprimento do cefalotórax entre 6 e $35 \mathrm{~mm}$. O crescimento do camarão sete-barbas, foi descrito pelas equações para os machos $\mathrm{Lc}=26,9\left(1-\mathrm{e}^{-0,7797 t}\right)$ e para as fêmeas $\mathrm{Lc}=37,6\left(1-\mathrm{e}^{-0,6328 t}\right)$. Já o crescimento em peso foi descrito pelas seguintes expressões: para machos $\mathrm{Wt}=11,7(1-\mathrm{e}$ o,7797t 2,7905 e para as fêmeas $\mathrm{Wt}=25, \mathrm{O}\left(1-\mathrm{e}^{-0,6328 t}\right) 2,5621$. Durante o período do estudo observou-se a ocorrência de fêmeas em todos os estádios gonadais. Os picos de desova ficaram evidenciados no período primavera/verão, enquanto que no outono/inverno observaram-se as maiores concentrações de fêmeas imaturas. $\mathrm{O}$ tamanho da primeira maturação das fêmeas foi estimado em 19,5 mm de comprimento do cefalotórax. Foi verificado que a atividade pesqueira está capturando na maior parte do ano fêmeas que não atingiram a maturidade sexual, situação que compromete a sustentabilidade da atividade.
\end{abstract}

Palavras-chave: Peneidae; pesca artesanal; crescimento; maturidade sexual; período reprodutivo.

\footnotetext{
Abstract. Biology and fishery of the seabob shrimp (Xiphopenaeus kroyeri, Heller 1862), in Balneário Barra do Sul, Santa Catarina, Brazil. The seabob shrimp fishery has shown a considerable reduction in production over the last years. Evaluating its extinction risk, the species has been classified as a "Data Deficient (DD)" one, therefore, this work aims to fulfill some of the gaps in the knowledge of seabob shrimp at a tradi-
} 
tional fishing area in the State of Santa Catarina, Brazil. There were 41 samples, where 14,873 individuals were observed and held. The females were predominant in most of the months, with a significant difference in the proportion comparing to the males. In males, the cephalothorax length varied between 6 and $28 \mathrm{~mm}$. Females, independent of the gonadal maturation stage, presented its cephalothorax length between 6 and $35 \mathrm{~mm}$. The growth of seabob shrimp can be described by the equation $\mathrm{Lc}=26.9\left(1-\mathrm{e}^{-0.7797 t}\right)$ for males, and $\mathrm{Lc}=37.6$ $\left(1-\mathrm{e}^{-0.6328 t}\right)$ for females. In weight, the equations to be used were: $\mathrm{Wt}=11.7\left(1-\mathrm{e}^{-0.7797 t}\right) 2.7905$ for males, and $\mathrm{Wt}=25 . \mathrm{O}\left(1-\mathrm{e}^{-0.6328 t}\right) 2.5621$ for females. During the study period, the occurrence of mature and also immature females has been observed. In the autumn and winter there were higher concentrations of immature females. Spawning peaks were evidenced in the spring and summer. The size of females at the first maturity was estimated at $19.5 \mathrm{~mm}$ by its cephalothorax length. As a conclusion, it was found that the fishery is, for the most part of the year, capturing females that have not reached sexual maturity, a situation that jeopardizes the sustainability of this activity.

Key words: Peneidae; artisanal fishing; growth; sexual maturity; recruitment; breeding period.

\section{Introdução}

O camarão sete-barbas (Xiphopenaeus kroyeri, Heller, 1862) apresenta ampla distribuição geográfica na costa Oeste do Oceano Atlântico, desde a Carolina do Norte (EUA) até o Brasil, na barra Norte da Ilha de Santa Catarina (Holthius, 1980; Tremel, 1968; Valentini et al., 1991). Entretanto, existem evidências de que se trata de duas espécies crípticas, com alta similaridade morfológica, possuindo ainda subpopulações distintas ao longo da costa brasileira (Gusmão et al., 2006).

Os camarões peneídeos apresentam quatro tipos de ciclos de vida: Tipo 1 - onde todas as fases ocorrem no ambiente estuarino; Tipo 2 - as pós-larvas e jovens habitam o estuário, enquanto os ovos, larvas e adultos utilizam o habitat marinho; Tipo 3 - onde todas as fases ocorrem no ambiente marinho; Tipo 4, onde todas as fases ocorrem em mar profundo (Dall et al.,1990). X. kroyeri não penetra normalmente em estuários, completando seu ciclo biológico em mar aberto, mas próximo à costa (Neiva \& Wise, 1963; Garcia \& Le Reste, 1981). Não há estratificação dos grupos etários, o estoque juvenil convive com o adulto na mesma área (Valentini et al., 1991). Entretanto, existem registros de ocorrência em zonas estatuarias (Holthius, 1959; 1980; Paiva et al., 1971; Oliveira, 1991), que estaria associada a elevada salinidade (Willians, 1965). Na região nordeste do Brasil, só não possui o ciclo de vida do Tipo 4, da classificação de Dall et al. (1990): Tipo 1 - ocorre na Baía do Iguape, sob influência do Rio Paraguaçu, nos arredores de Maragogipe e Santiago do Iguape, na região do Recôncavo Baiano; Tipo 2 - ocorre no estuário do Rio Sergipe, em Aracaju; Tipo 3 - é o ciclo mais comum ao longo da costa do nordeste (Santos, et al., 2006; Santos, et al., 2013).

$X$. kroyeri habita em fundo de areia e lama até os 118 metros de profundidade (Coelho, 1980). Porém, é mais abundante até 27 metros (FAO, 1978). Nas Guianas Francesas os melhores rendimentos ocorrem entre 20 e 24 metros (Oliveira, 1991), situação semelhante àquela observada na Armação do Itapocoroy, Penha, SC, onde as maiores concentrações e comprimento de adultos foram observados nos 20 metros (Branco et al., 2013). As maiores abundâncias são observadas em águas de salinidade mais elevada, sendo a espécie pescada pela frota de arrasto, tanto industrial como artesanal que constituí a principal modalidade de captura na costa brasileira (Dias-Neto, 2011; Heckler et al., 2013). 
Nas regiões Sudeste-Sul do país, as maiores capturas foram obtidas no período de 1972 a 1982, com volume anual desembarcado, oscilando entre 9.830 e $15.591 \mathrm{t}$ em 1981, com rendimento máximo sustentável estimado em cerca de 14.ooot. Após esse período observou-se uma contínua diminuição dos desembarques, sempre atingindo menos de 10.ooot. A captura total no Estado de Santa Catarina em 1993 e 1994, período de amostragem desta pesquisa, foi respectivamente de 6.334 e 8.706t. (Valentini et al.,1991; Dias-Neto, 2011). Em geral, as capturas monitoradas a partir de 2000 apresentam um rendimento anual inferior a 40\% do melhor rendimento entre 19652007 (15.591t) (Dias-Neto, 2011; IBAMA, 2009). Contudo, dados dos desembarques do litoral de São Paulo entre 2000 e 2011 indicam valores de CPUE $(\mathrm{kg} / \mathrm{h}$ ) estáveis, que pode alterar a avaliação sobre o recurso (Mendonça et al., 2013).

Apesar das recomendações para a redução do esforço pelo controle da frota e estabelecimento de um período de defeso no SudesteSul, o rendimento máximo sustentável foi estimado em 7.000 t entre 1990-1999 (D'Incao et al., 2002; Dias-Neto, 2011).

$\mathrm{Na}$ recente avaliação do risco de extinção da espécie, foi incluída na categoria de "Dados Insuficientes - DD” (IUCN, 2014; ICMBio, 2015), pela falta de informações adequadas, principalmente de estatística pesqueira desde 2008 da frota artesanal nacional. Portanto, ainda não são conhecidos os níveis de captura e desembarque do camarão setebarbas na costa brasileira.

Durante o período amostral (1993-94) havia no Balneário Barra do Sul uma frota de 42 embarcações de pequeno porte, com menos de $10 \mathrm{AB}$ (arqueação bruta), que operava na área Norte da Ilha dos Remédios, no período das quatro às 14 horas, entre profundidades de 12 a 15 metros, efetuando de dois a três lances com duração média de 02 horas. Atualmente (2014), de acordo com a Federação dos Pesca- dores do Estado de Santa Catarina, 104 embarcações possuem licença para a captura de camarão. Contudo, esse número de embarcações com licença de pesca, talvez não reflita de fato a quantidade de embarcações que operam na área, sendo necessários estudos que dimensionem a atividade.

Apesar de apresentar dados coletados há duas décadas, esse trabalho preenche uma lacuna no conhecimento, aportando informações sobre a proporção entre os sexos, maturidade gonadal e curvas de crescimento para machos e fêmeas do camarão sete-barbas no litoral do Balneário de Barra do Sul, tradicional área de pesca no litoral catarinense, contribuindo na gestão e manejo deste importante recurso pesqueiro.

\section{Materiais e Métodos}

As amostragens foram realizadas a partir de duas coletas mensais entre fevereiro de 1993 a novembro de 1994, no Balneário Barra do Sul, SC (Figura 1). Nos meses de abri e setembro de 1993 não houve amostragem biológica devido a problemas de logística que impediram o trabalho no campo.

Da captura total desembarcada, portanto previamente separada da fauna acompanhante, oriunda de uma mesma embarcação (escolhida da frota camaroneira motorizada) e mesmo petrecho de pesca, era obtida uma amostra aleatória de $2 \mathrm{~kg}$ de camarão setebarbas, antes da seleção feita pelo pescador para a venda. Os exemplares foram acondicionados em uma badeja plástica e colocados dentro de um isopor com gelo, tendo posteriormente sido transportados até o laboratório, onde após serem lavados, foram conservados sob-refrigeração, até o dia da amostragem.

Os camarões foram separados por sexo, ou seja, presença de télico nas fêmeas e de petasma nos machos. Das fêmeas determinou-se o estádio de maturação gonadal por observação macroscópica, através da transparência do exo- 


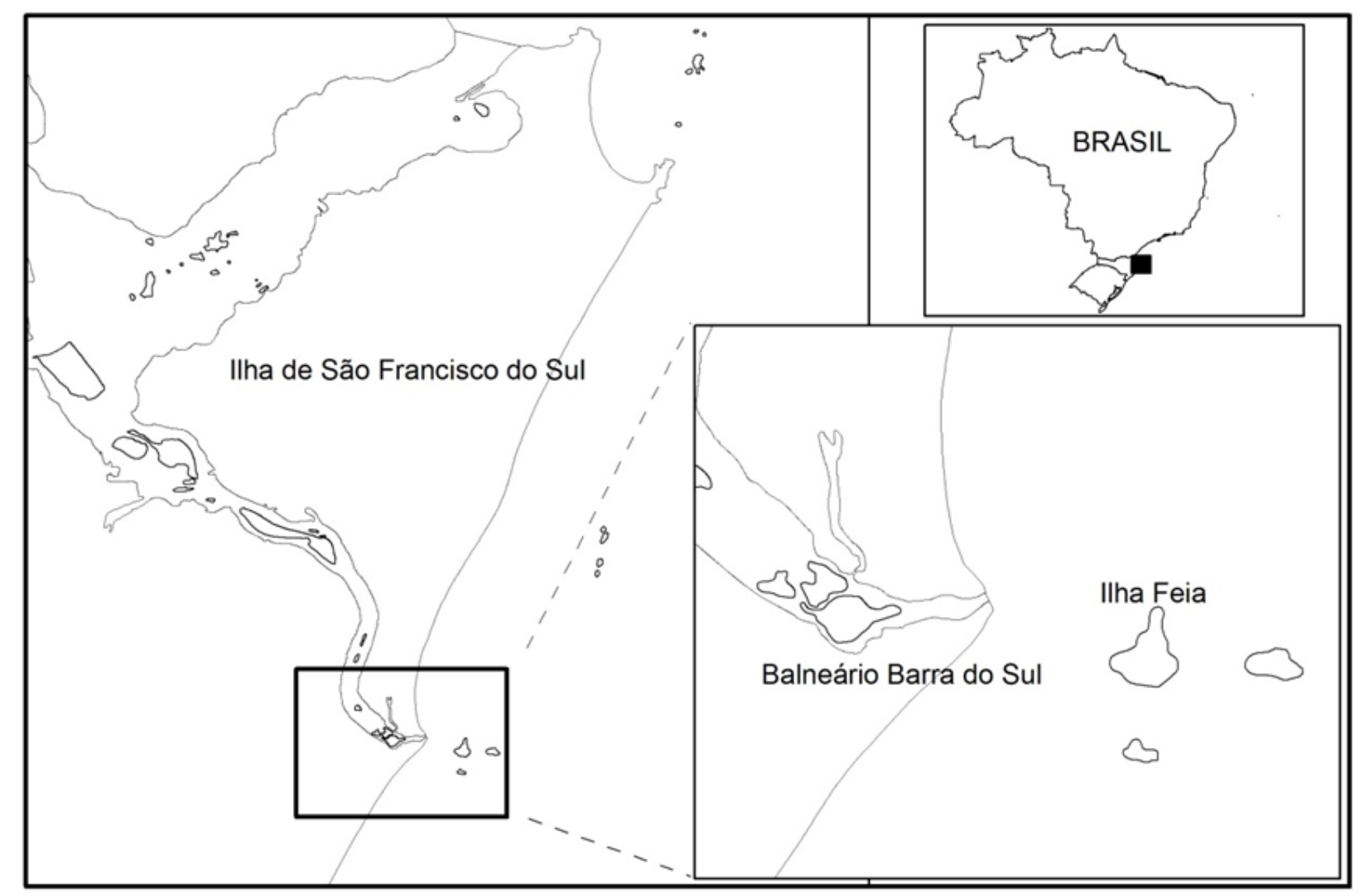

Figura 1. Localização do Balneário Barra do Sul, Santa Catarina, Brasil.

esqueleto, na região dorsal. As gônadas foram classificadas em imaturas (estádio I) e maturas (estádios II - em maturação, III - maturas e IV - desovando) (Alvez \& Rodrigues, 1977). As poucas fêmeas observadas em estádio de desova (estádio IV) foram agrupadas no estádio III.

Devido à fragilidade da extremidade anterior do rostro, não optou-se pelo comprimento total, portanto, a biometria foi do comprimento do cefalotórax (Lc), ou seja, medida entre o ângulo orbital e a extremidade livre, utilizando-se um paquímetro com precisão de $0,1 \mathrm{~mm}$.

A proporção sexual, a cada mês, foi obtida pela razão entre o número total de machos/fêmeas amostrados, sendo a diferença entre os sexos verificada pelo teste de ajustamento (distribuição Qui-quadrado; $\chi^{2}$ crit $=3,84$ ) com nível de significância de $5 \%$.

A época do recrutamento foi determina- da pela análise da variação temporal do comprimento médio do cefalotórax, considerando que a redução do tamanho individual, em alguns meses, está relacionada com a integração de uma grande quantidade de indivíduos jovens ao estoque capturável.

O tamanho médio de primeira maturação, quando $50 \%$ dos indivíduos entram ativamente na fase de maturação gonadal, foi estimado para as fêmeas de acordo com Vazzoler (1981).

As curvas de crescimento do comprimento do cefalotórax (Lc) foram obtidas pelo método da distribuição de frequência de comprimento para os sexos separados, bimestralmente (Santos, 1978). Foi utilizada a expressão de Bertalanffy (1938): $\mathrm{Lc}_{\mathrm{t}}=\mathrm{L} \infty$ [1-e $\left.\mathrm{-k}^{-\mathrm{t}\left(\mathrm{t}_{\mathrm{o}}\right)}\right]$, onde "Lct" = comprimento médio do cefalotórax na idade "t"; "Lo"= comprimento máximo médio do cefalotórax; "e"= base dos logaritmos natu- 
rais; " $K "=$ a constante relacionada com a taxa de crescimento; " $\mathrm{t}_{\mathrm{o}}$ "= idade teórica quando o seu comprimento é igual a zero; "t"= idade considerada.

A validade de expressão de Bertalanffy para a espécie foi comprovada pela transformação de Ford-Walford (1946) e os parâmetros estimados conforme Santos (1978).

As curvas de crescimento em peso foram obtidas pelo método dedutivo (Santos op. cit), após o conhecimento da relação peso/ comprimento do cefalotórax para machos e fêmeas, a partir da expressão: $\mathrm{W}_{\mathrm{t}}=\mathrm{W} \infty\left[1-\mathrm{e}^{-\mathrm{k}(\mathrm{t}-}\right.$ $\left.\left.\mathrm{t}_{\mathrm{o}}\right)\right] \theta$, onde " $\mathrm{W}_{\mathrm{t}}$ " = peso na idade " $\mathrm{t} "$; "Wo"= peso máximo médio; "e"= base dos logaritmos naturais; $\mathrm{e}$ " $\theta$ " = constante relacionada com o crescimento da espécie. Para X. kroyeri, cujo comprimento é desprezível ao nascer, assumese que " $\mathrm{t}_{0}$ " seja igual a zero.

\section{Resultados e Discussão}

Entre fevereiro de 1993 e novembro de 1994, foram realizadas 41 amostragens e mensurados 14.873 indivíduos, sendo 7.172 machos e 7.701 fêmeas (Tabela 1), com predomínio das fêmeas em 13 dos 20 meses de estudo. Estes dados corroboram outros estudos que observaram até 70\% de fêmeas (Tremel, 1968).

A análise do teste $\chi^{2}$, para comparação mensal da proporção de machos e fêmeas indicou a existência de predomínio dos machos, nos meses de março/93, janeiro e abril/94, en-

Tabela 1. Valores mensais e por sexo, da frequência absoluta, amplitude de comprimento de cefalotórax (mm), tamanho médio, número de indivíduos por quilo e teste de aderência $\left(\mathrm{X}^{2}\right)$ dos camarões sete-barbas capturados entre fevereiro de 1993 a novembro de 1994, no Balneário Barra do Sul, SC.

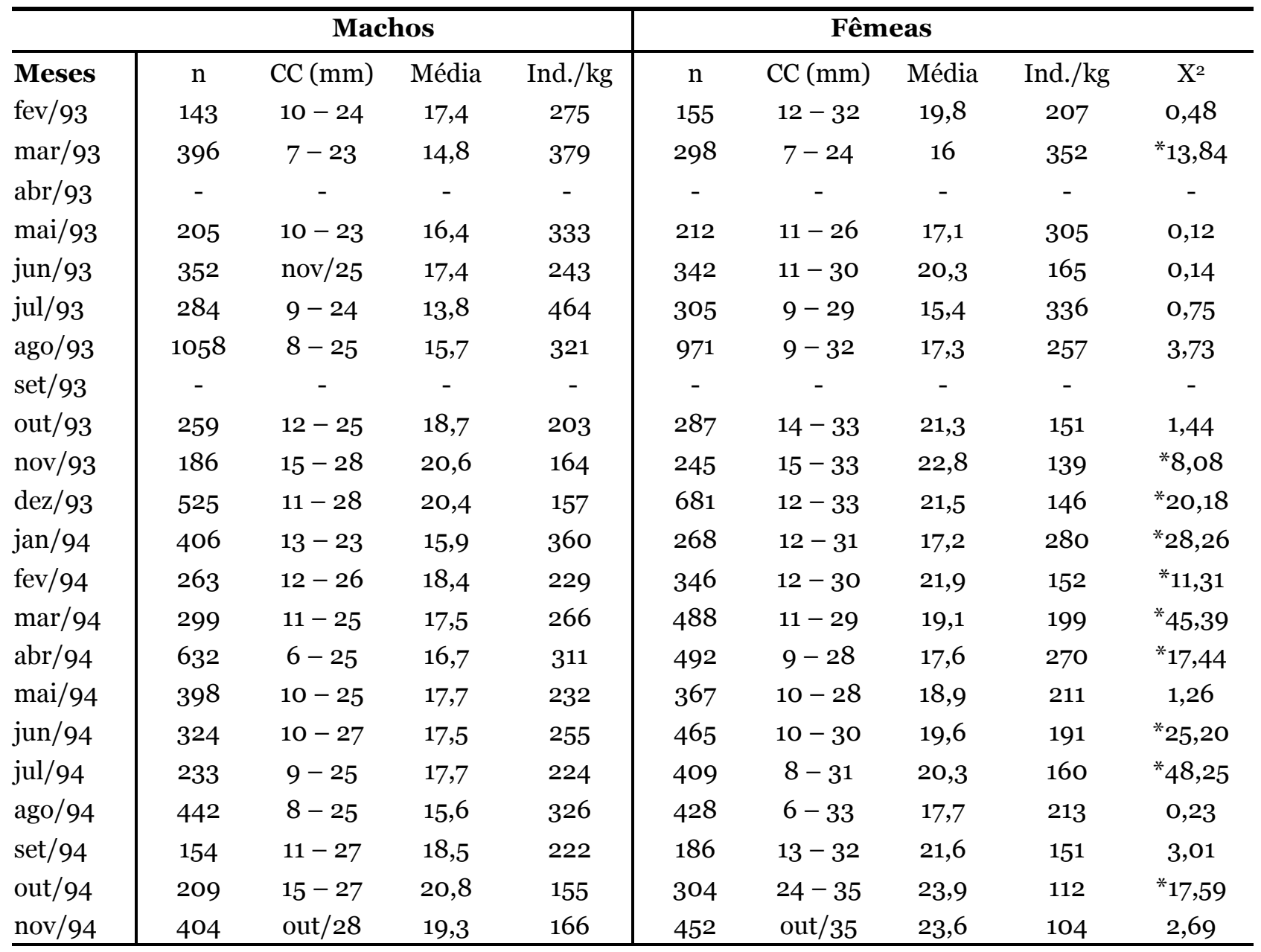

* Diferença significativa na proporção entre os sexos. 
quanto as fêmeas foram mais significativas em novembro e dezembro/93 e nos meses de fevereiro, março, junho, julho e outubro/94. No restante dos meses não foi observada diferença estatisticamente significante nas proporções de machos e fêmeas (Tabela 1).

Nos machos o intervalo de comprimento do cefalotórax variou entre 6 e $28 \mathrm{~mm}$, com média anual de $17,5 \mathrm{~mm}$, tendo a média mensal variado entre 13,8 (julho/93) e 20,8 mm (outubro/94). Já as fêmeas, independente do estádio de maturação gonadal, apresentaram intervalo de comprimento do cefalotórax entre $6 \mathrm{~mm}$ e $35 \mathrm{~mm}$, porém, com média anual de 19,6 mm, com mínima mensal $(15,4 \mathrm{~mm})$ em julho/93 e máxima (23,9 mm) em outubro/94 (Tabela 1). As informações deste trabalho coincidem com a literatura disponível sobre os peneídeos, que afirma a existência de comprimento médio mais elevado nas fêmeas em relação aos machos (Dall et al., 1990).

O número de camarões sete-barbas necessários para formar um quilograma é utilizado pelos pescadores como um indicador do tamanho comercial da espécie. Assim, quanto menor for o número por quilo, mais uniforme será a composição de tamanho da captura, evidenciando um bom porte dos indivíduos, portanto, a pescaria fica economicamente mais viável. Em Balneário Barra do Sul, no período estudado, foi observada uma média anual de 264 machos/kg e 206 fêmeas $/ \mathrm{kg}$, sendo os meses de novembro e dezembro/93 e outubro e novembro/94 os meses com menor número de machos e fêmeas por $\mathrm{kg}$ de camarão setebarbas capturado (Tabela 1).

O aumento do número de indivíduos de pequeno porte em uma área de pesca ocorre provavelmente devido à intensificação do processo de recrutamento. Isso pode ser verificado pela redução do comprimento médio do estoque explotado e pelo aumento no número de espécimes por quilo. Considerando estes aspectos, verifica-se que no Balneário Barra do Sul o pico do recrutamento ocorreu durante o perío- do amostral nos meses de julho/1993 e janeiro/ 1994 (Tabela 1).

A ocorrência de mais de um pico de recrutamento ou a entrada contínua de jovens na população adulta já foi observada em algumas pesquisas sobre a biologia dos camarões setebarbas (Santos e Ivo, 2000; Santos et al. 2003; Santos et al., 2007; Branco et al., 2013; Santos et al. 2013; Couto et al., 2013; Martins et al., 2013). Esta situação torna praticamente impossível a proteção integral do recrutamento a partir do estabelecimento de períodos de defeso. Atualmente o período de defeso do camarão sete-barbas é definido pela Instrução Normativa IBAMA $189 / 2008$ que proibi a pesca de $1^{\circ}$ de março até 31 de maio. Este período, contudo, leva em consideração principalmente a reprodução do camarão rosa (Farfantepenaeus paulensis, $F$. brasiliensis) e a eficácia na aplicação da norma, visto a dificuldade de fiscalização de vários períodos de proibição de captura.

Além da dificuldade de proteção dos recrutas pelo estabelecimento de períodos de defeso, no Balneário Barra do Sul houve o aumento de mais de $100 \%$ no número de embarcações voltadas a esta pescaria em 20 anos. Em 1993-94 eram 42 embarcações, já em 2014, de acordo com a Federação dos Pescadores do Estado de Santa Catarina, são 104 embarcações com licença para a captura de camarão.

Durante o período estudado observouse a ocorrência tanto de fêmeas imaturas (estádio I) como de maturas (estádios II, III e IV). A presença de fêmeas maturas em todo o período apresentou variações, mínima de $7 \%$ em julho/93 e máxima de 89\% em outubro/94. Verificou-se também a existência de picos (mais de 50\%) nos meses de fevereiro, novembro e dezembro de 93 e fevereiro, outubro e novembro de 94 (Figura 2). Estes períodos coincidem com um menor número de fêmeas por quilo. As fêmeas imaturas (estádio I) mantiveram uma participação mínima de $11 \%$ em outubro/94 e máxima de 93\% em julho/93, confirmando a coexistência de camarões jovens, a- 
dultos e ausência de estratificação populacional.

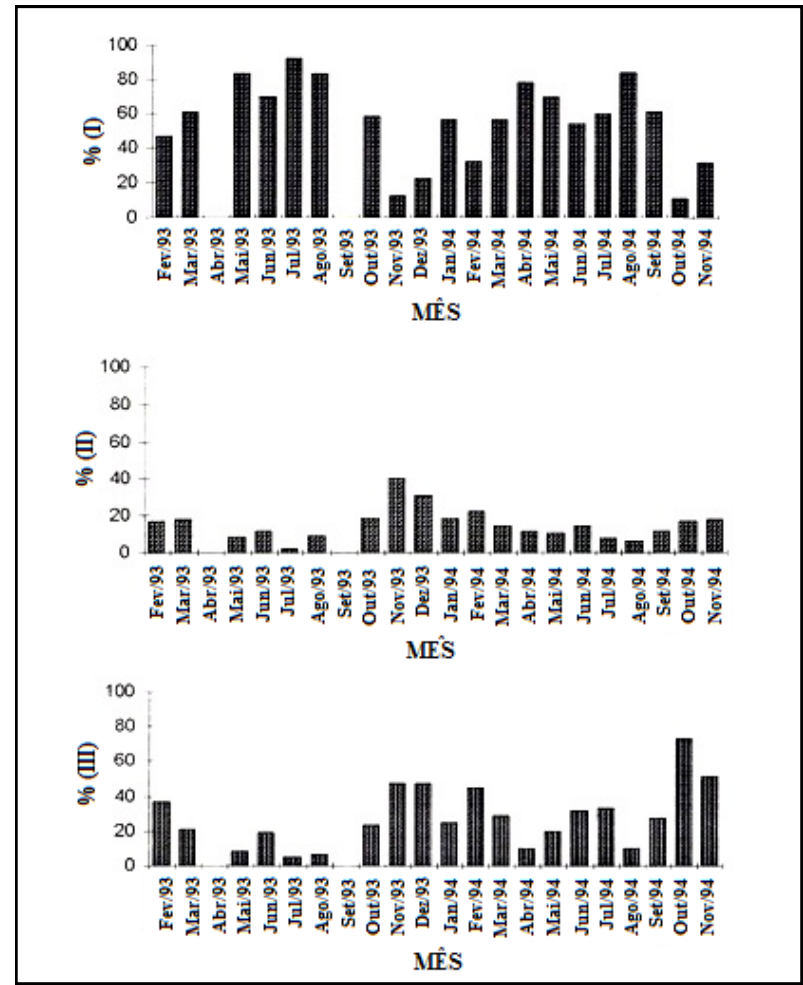

Figura 2. Percentual de fêmeas imaturas (estádio I) e maturas (estádios II, III e IV) de $\mathrm{Xi-}$ phopenaeus kroyeri (Heller, 1862) coletadas entre fevereiro de 1993 e novembro de 1994 no Balneário Barra do Sul, SC.

Os picos de fêmeas ficaram evidenciados no período primavera/verão, enquanto que no outono/inverno observaram-se as maiores concentrações de fêmeas imaturas (Figura 3). Resultados semelhantes foram obtidos em outras localidades de Santa Catarina, Paraná, São Paulo, Rio de Janeiro e Pernambuco (Amado, 1961; Neiva \& Wise, 1963; Tremel, 1968; Coelho \& Santos, 1983; Santos \& Ivo, 2000; Fernandes et al., 2011). Enquanto que, na Guiana Francesa, as fêmeas em maturação foram mais frequentes em abril, julho, agosto, outubro e novembro (Oliveira, 1991).

O tamanho da primeira maturação das fêmeas foi estimado em 19,5 mm de comprimento do cefalotórax, o que corresponde a aproximadamente $98 \mathrm{~mm}$ de comprimento total (Figura 4). Esse valor quando comparado às distribuições mensais de frequência do comprimento, indica que a atividade pesqueira está capturando na maior parte do ano, fêmeas que não atingiram a maturidade sexual (Figura 5). Considerando ainda, que os machos atingem comprimento máximo médio menor que as fêmeas, pode-se supor que a captura de imaturos também seja predominante. Desta forma, a pesca incide indistintamente sobre jovens e adultos de ambos os sexos na população, entre 6 e $35 \mathrm{~mm}$ de comprimento do cefalotórax.

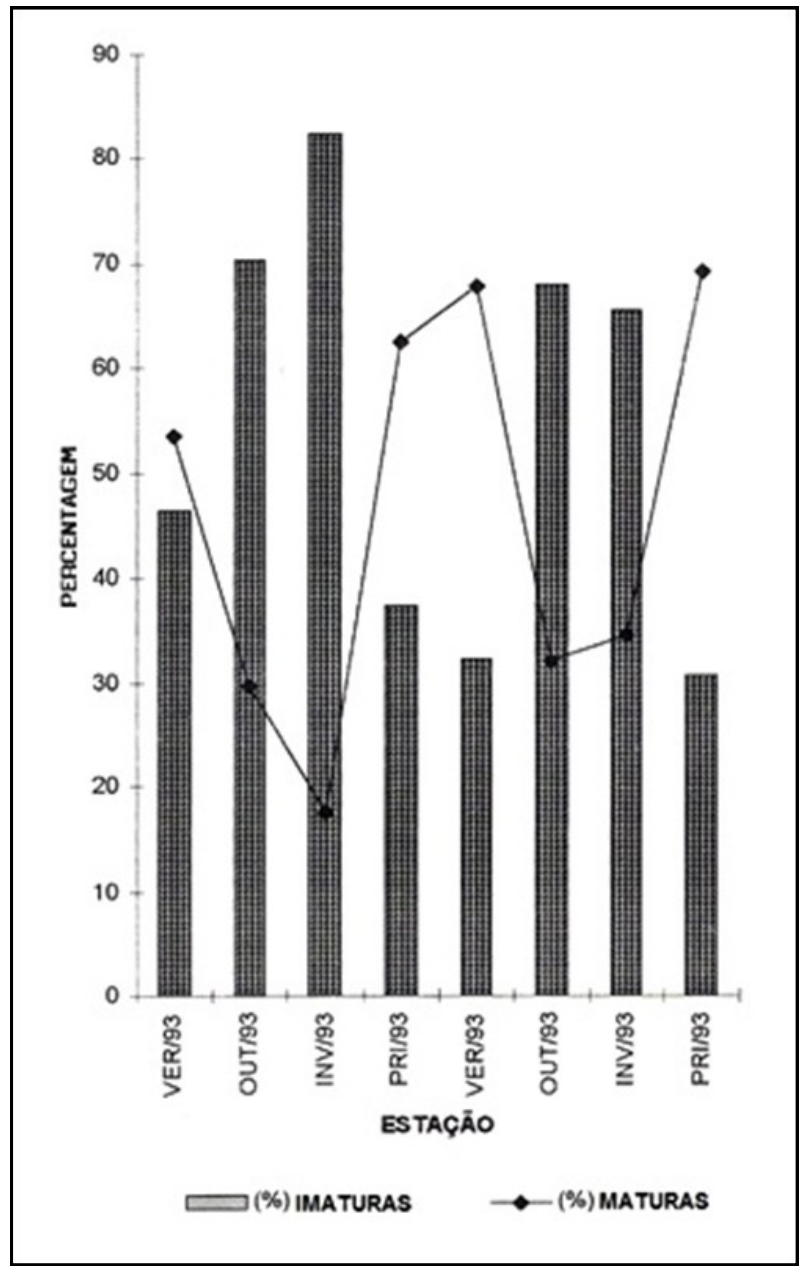

Figura 3. Percentual de fêmeas imaturas (estádio I) e maturas (estádios II, III e IV) de $X i$ phopenaeus kroyeri (Heller, 1862) coletadas entre fevereiro de 1993 e novembro de 1994 no Balneário Barra do Sul, SC, distribuídas ao longo das estações do ano.

No litoral paulista a primeira maturação gonadal foi estimada em $62 \mathrm{~mm}$ (machos) e $71 \mathrm{~mm}$ (fêmeas) de comprimento total (Severino-Rodrigues et al., 1993); no norte do Rio de Janeiro, em $66 \mathrm{~mm}$ comprimento total (12 mm de cefalotórax) para machos e $109 \mathrm{~mm}$ de comprimento total (22 $\mathrm{mm}$ de cefalotórax) 
nas fêmeas (Fernandes et al., 2011).

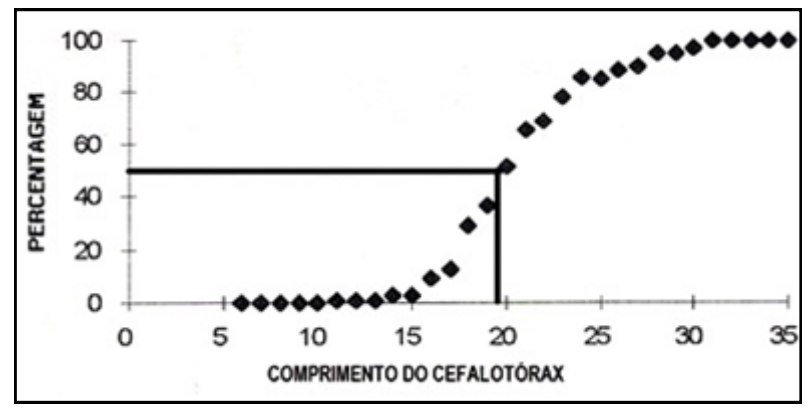

Figura 4. Distribuição da frequência relativa de fêmeas maturas de Xiphopenaeus kroyeri (Heller, 1862) coletadas entre fevereiro de 1993 e novembro de 1994 no Balneário Barra do Sul, SC, por classe de comprimento do cefalotórax ( $\mathrm{mm}$ ).

A idade de primeira reprodução dos camarões coletados em Balneário Barra do Sul foi estimada em 14 meses; no litoral do Paraná ficou em torno de 18 meses (Amado, 1978) e 12 meses na região nordeste de São Paulo (GraçaLopes et al., 2007).

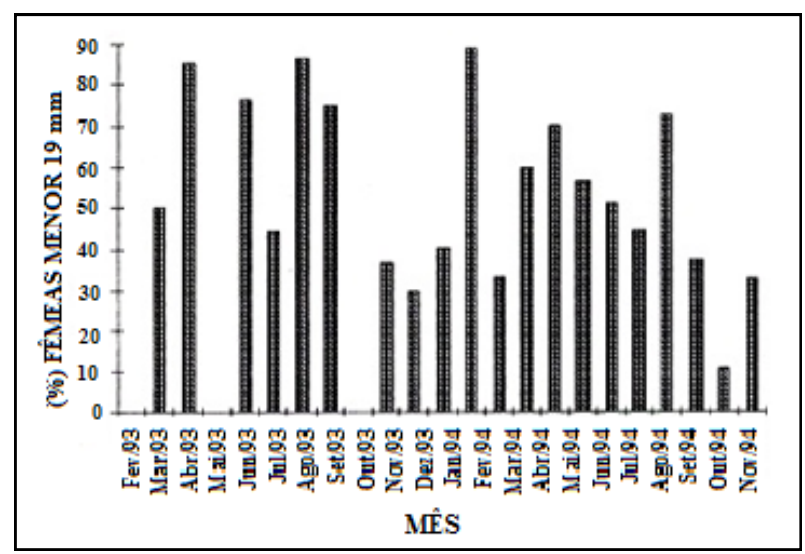

Figura 5. Distribuição da frequência relativa de fêmeas com tamanho inferior ao da primeira maturação sexual (Lc 19,5 mm) de Xiphopenaeus kroyeri (Heller, 1862) coletadas entre fevereiro de 1993 e novembro de 1994 no Balneário Barra do Sul, SC.

As diferenças registradas entre os tamanhos e idades da primeira maturação ao longo da costa brasileira, além de refletir as condições ambientais locais que as populações estão submetidas, podem indicar esforços de pesca diferenciados.

Nascimento \& Poli (1985), trabalhando com a população $X$. kroyeri da Baía de Tijucas
(SC), ajustaram somente uma curva de comprimento total, sugerindo ausência de dimorfismo sexual quanto a este parâmetro. Entretanto, quando se comparam os comprimentos e pesos máximos médios obtidos neste trabalho, na população do Balneário Barra do Sul, torna-se evidente a existência de dimorfismo sexual e, portanto, a necessidade da aplicação de equações distintas. A partir da análise das modas calculadas ao longo do período de coleta (Figura 6), foram estimados os parâmetros das curvas de crescimento, para machos: $\mathrm{Lc}=26,9$ $\left(1-\mathrm{e}^{-0,7797 t}\right)$ e fêmeas: $\mathrm{Lc}=37,6\left(1-\mathrm{e}^{-0,6328 t}\right)($ Figura 7).
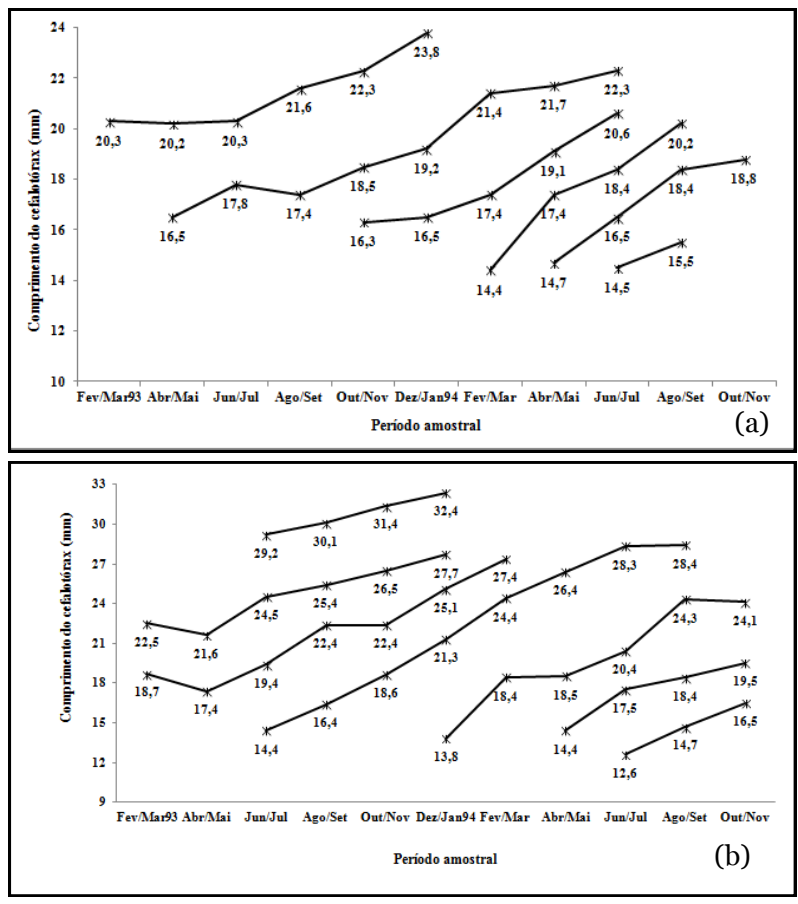

Figura 6. Modas bimestrais do comprimento do cefalotórax de machos (a) e fêmeas (b) de Xiphopenaeus kroyeri (Heller, 1862) coletados entre fevereiro de 1993 e novembro de 1994 no Balneário Barra do Sul, SC.

A espécie apresenta uma taxa de crescimento elevada, com valores de $\mathrm{k}=0,7797$ para machos e k=0,6328 para fêmeas, sendo estimado, de acordo com as curvas de crescimento obtidas, que o comprimento máximo médio do cefalotórax (Lo) é atingido em torno de 30 meses de idade.

As curvas de crescimento obtidas para $X$. kroyeri em algumas localidades do litoral 
brasileiro foram estimadas com base no comprimento total (Lt). Os menores comprimentos máximos médios foram observados em Martinhos (PR), 12,1 cm para machos e 14,9 cm para fêmeas (Amado, 1978). Enquanto que, na Baía de Santos (SP), os valores foram 14,4 cm para machos e 16,2 cm para fêmeas (Santos et al., 1969).
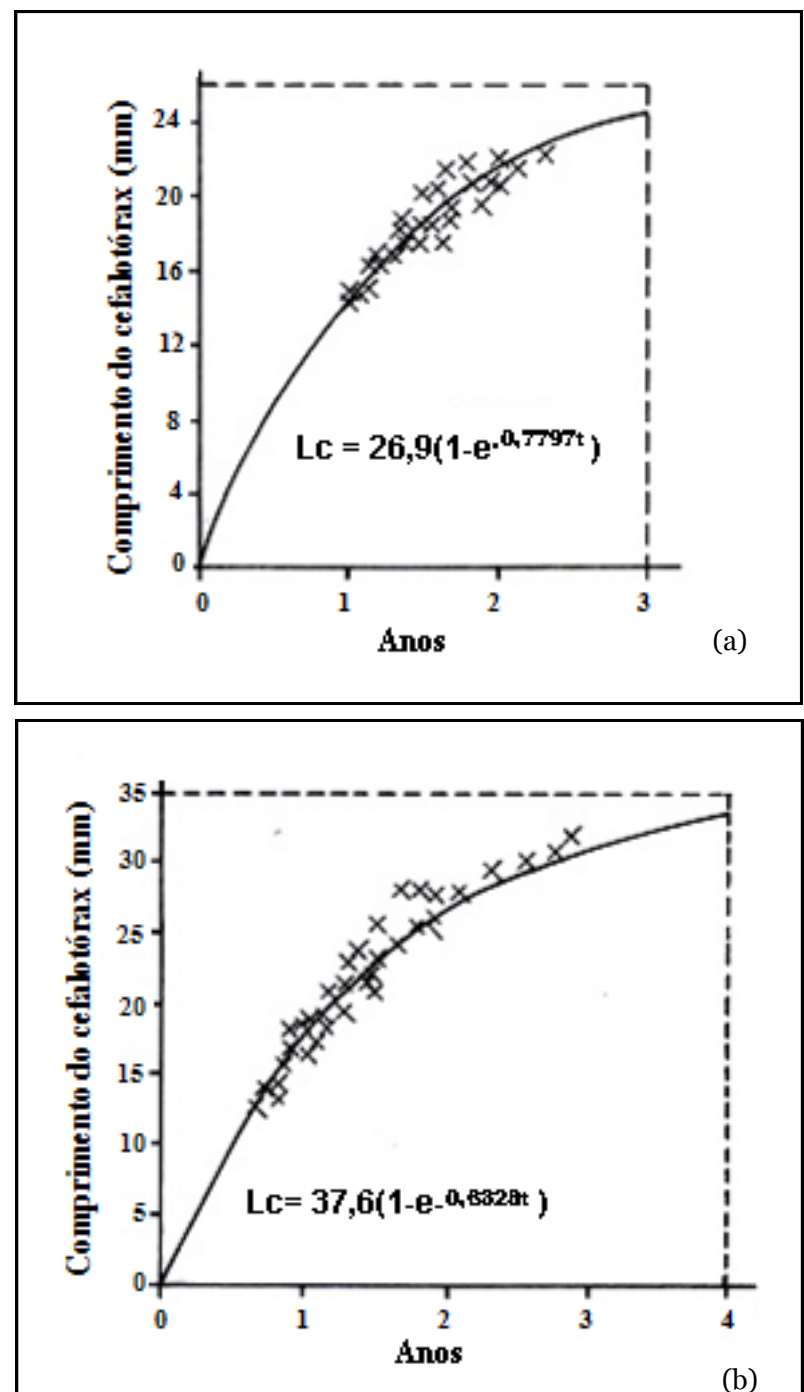

Figura 7. Curva de crescimento do comprimento do cefalotórax (mm) de machos (a) e fêmeas (b) de Xiphopenaeus kroyeri (Heller, 1862) coletados entre fevereiro de 1993 e novembro de 1994 no Balneário Barra do Sul, SC.

Já o crescimento em peso foi descrito pelas seguintes expressões: para machos $\mathrm{Wt}=11,7\left(1-\mathrm{e}^{-0,7797 t}\right) 2,7905$ e para as fêmeas $\mathrm{Wt}=25, \mathrm{O}\left(1-\mathrm{e}^{-0,6328 \mathrm{t}}\right) 2,5621$ (Figura 8).

Os machos atingem em uma mesma classe de comprimento, em média, menor peso que as fêmeas. Situação semelhante foi registrada no litoral do Paraná (Branco et al., 1994).

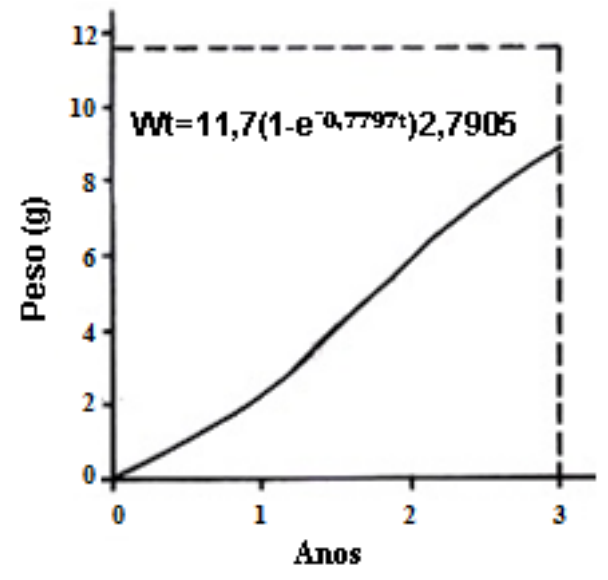

(a)

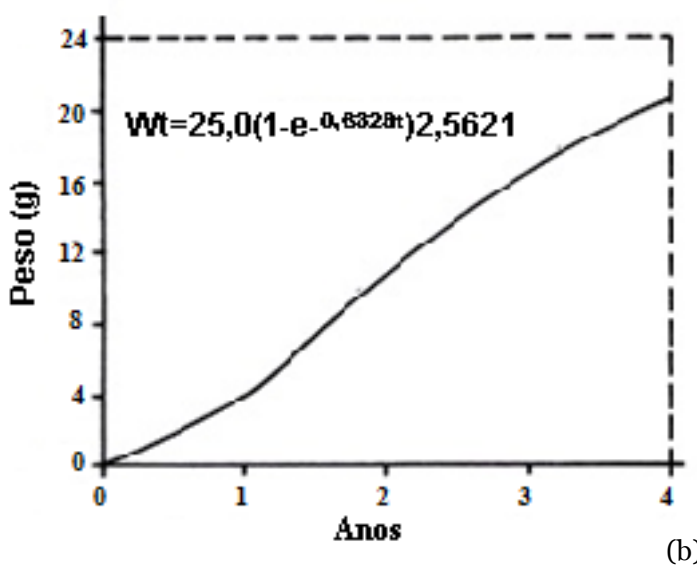

(b)

Figura 8. Curva de crescimento do peso (g) de machos (a) e fêmeas (b) de Xiphopenaeus kroyeri (Heller, 1862) coletados entre fevereiro de 1993 e novembro de 1994 no Balneário Barra do Sul, SC.

\section{Conclusões}

- Dos 20 meses em que ocorreu amostragem, em 10 não foi observada diferença significante nas proporções de machos e fêmeas.

- O pico do recrutamento ocorreu nos meses de julho/1993 (inverno) e janeiro/1994 (verão).

- A maturidade das fêmeas foi estimada em 19,5 mm de comprimento do cefalotórax, aproximadamente $98 \mathrm{~mm}$ de comprimento total, sendo a idade de primeira reprodução 
estimada em 14 meses.

- A atividade pesqueira no Balneário Barra do Sul está capturando na maior parte do ano fêmeas que não atingiram a maturidade sexual.

- A ocorrência de 2 picos de recrutamento dificulta a proteção integral dos recrutas a partir do estabelecimento de períodos de defeso, sendo necessárias outras medidas de ordenamento, em especial aquelas voltadas à diminuição do esforço, visto que em 20 anos (1994 - 2014) houve um aumento superior à 100\% no número de embarcações voltadas a esta pescaria no Balneário Barra do Sul.

\section{Agradecimentos}

Aos colegas do CEPSUL pelo apoio durante as amostragens e preparação do manuscrito, em especial Elisabeth Michelleti e Eloisa Pinto Vizuete.

Aos revisores anônimos da Revista CEPSUL pela contribuição fundamental na publicação deste artigo.

\section{Referências Bibliográficas}

ALVES, M. I. M. \& M. M. RODRIGUES. 1977. Aspectos da reprodução do camarão-setebarbas Xiphopenaeus kroyeri (HELLER) (Decapoda: Macrura), na Costa do Estado do Ceará. Arq. Ciên. Mar., 17(1): 29-35.

AMADO, M. A. P. M. 1978. Estudos biológicos do Xiphopenaeus kroyeri (Heller, 1862), camarão sete-barbas (Crustacea, Penaeidae) de Matinhos, PR. 10op. (Dissertação de Mestrado. Universidade Federal do Paraná, UFPR).

BERTALANFFY, L. V. 1938. A Quantitative theory of organic growth. Hum. Biol., 1(2): 181213.

BRANCO, J. O, M. J. LUNARDON-BRANCO \& A. de FINIS. 1994. Crescimento de Xiphopenaeus kroyeri (Heller, 1862), (Crutacea: Natantia: Penaeidade) da região de Matinhos, Paraná, Brasil. Arq. Biol. Tecnol., 37(1): 1-8.
BRANCO, J. O., L. R. SANTOS, E. BARBIERI, M. do C. F. SANTOS \& J. L. RODRIGUES FILHO. 2013. Distribuição espaço-temporal das capturas do camarão sete-barbas na Armação do Itapocoroy, Penha, SC. Bol. Inst. Pesca, 39 (3): $237-250$.

COELHO, P. A., M. RAMOS-PORTO \& M. L. KOENING. 1980. Biogeografia e bionomia dos crustáceos do litoral equatorial brasileiro. Trab. Oceanogr. UFPE, 15: 7-138.

COUTO, E. C. G., F. J. GuimarÃES, C. A. M. OLIVEIRA, R. O. R. VASQUES, J. B. B. S. SANTOS LOPES. 2013. O camarão setebarbas na Bahia: aspectos da sua pesca e biologia. Bol. Inst. Pesca, 39: 263-282.

DALL, W., B. J. HILL, P. C. ROTHLISBERG \& D. J. STAPLES, 1990. The biology of Penaeidae. In BLAXTER, J. H. S. \& A. J. SOUTHWARD. Advances in Marine Biology, v. 27. Academic Press, San Diego. 489p.

DIAS-NETO, J. (Org.). 2011. Proposta de Plano Nacional de Gestão para o uso sustentável de Camarões marinhos do Brasil. Brasília, DF, IBAMA, 242p.

D'INCAO, F., H. VALENTINI \& L. F. RODRIGUES. 2002. Avaliação da pesca de camarões nas regiões sudeste e sul do Brasil 1965-1999. Atlântica, 24(2): 103-116.

FAO - Food and Agriculture Organization of the United Nations. 1978. Species identification sheets for fishery purposes, Western Central Atlantic (Fishery Area 31). FISCHER, W. (Ed.) FAO, Rome, v.6. sp.

FERNANDES, L. P., A. C. SILVA, L. P. JARDIM, K. A. KEUNECKE \& A. P. M. DI BENEDITTO. 2011. Growth and Recruitment of the Atlantic Seabob Shrimp, Xiphopenaeus kroyeri (Heller, 1862) (Decapoda, Penaeidae), on the Coast of Rio de Janeiro, Southeastern Brazil. Crustaceana, 84(12-13): 14651480 .

GARCIA, S. \& L. LE RESTE. 1981 Life Cycles, Dynamics, Exploitation and Management of Coastal Penaeid Shrimp Stocks. FAO Fish. Tech. Pap., (203). 210 p. 
GRAÇA-LOPES, R., E. P. SANTOS, E. SEVERINO -RODRIGUES, F. M. S. BRAGA \& A. PUZZI. 2007. Aportes ao conhecimento da biologia e da pesca do camarão sete-barbas Xiphopenaeus kroyeri (Heller, 1862) no Litoral do Estado de São Paulo, Brasil. Bol. Inst. Pesca, 33 (1): $63-84$.

GUSMÃO, J., C. LAZOSKI, F. A. MONTEIRO \& A. M. SOLÉ-CAVA. 2006. Cryptic species and population structuring of the Atlantic and Pacific seabob shrimp species, Xiphopenaeus kroyeri and Xiphopenaeus riveti. Marine Biology, 149: 491-502.

HECKLER, G. S., S. M. SIMÕES, M. LOPES, F. Z. ZARA \& R. C. COSTA. 2013. Biologia populacional e reprodutiva do camarão-sete-barbas na Baía de Santos, São Paulo. Bol. Inst. Pesca, 39: 283-297.

HOLTHUS, L. B. 1980. Shrimp and prawns of the world. An Annoted Catalogue of Species of Interest to Fischeries. FAO Fischeries Synopsis, 1(125), 271p.

IBAMA, 2009. Estatísticas da pesca 2007: Brasil e grandes regiões e unidades da federação. Brasília, DF, IBAMA, 113p.

ICMBio - Instituto Chico Mendes de Conservação da Biodiversidade. 2015. Lista de Espécies Quase Ameaçadas ou com Dados Insuficientes. Disponível em: http:// www.icmbio.gov.br/portal/biodiversidade/ fauna-brasileira/lista-de-especies-dadosinsuficientes.html. Acesso: 20 set. 2015.

IUCN Standards and Petitions Subcommittee. 2014. Guidelines for Using the IUCN Red List Categories and Criteria. Version 11. Prepared by the Standards and Petitions Subcommittee. Disponível em: http:// www.iucnredlist.org/ documents/ RedListGuidelines.pdf. Acesso em: 10 jan. 2015.

MARTINS, A. S., H. T. PINHEIRO \& N. O. LEITE JUNIOR. 2013. Biologia reprodutiva do camarão-sete-barbas no litoral centro-sul e sul do Espírito Santo, Brasil. Bol. Inst. Pesca, 39: 205-215.

MENDONÇA, J. T., R. da GRAÇA-LOPES \& V. G. DE AZEVEDO. 2013. Estudo da CPUE da pesca paulista dirigida ao camarão setebarbas entre 2000 e 2011. Bol. Inst. Pesca, 39 (3): $251-261$.

NASCIMENTO, P. A. M. \& C. R. POLI. 1985. Curva de crescimento do camarão-sete-barbas, Xiphopenaeus kroyeri (Heller, 1862), na Baia de Tijucas - Santa Catarina. In: ANAIS DO I SEMINÁRIO SOBRE CIÊNCIAS DO MAR DA UFSC - Florianópolis: p. 37- 41.

NEIVA, G. S. \& J. P. WISE. 1963. The biology and fishery of the sea bob shrimp of Santos Bay, Brazil. Process of Gulf Caribbean Fisheries Institute, Miami, 16: 131-139.

OLIVEIRA, J. E. L. 1991. Biologie et dynamique dês populations de la crevete Xiphopenaeus kroyeri (Heller, 1862), em Guyane Française. Paris. 190p. (Tese de Doutorado. Université Pierre et Marie Curie).

PAIVA, M. P., R. C. F. BEZERRA, \& A. A. FONTELES FILHO. 1971. Tentativa de avaliação dos recursos pesqueiros do Nordeste Brasileiro. Arq. Cienc. Mar., 1(11): 1-15.

SANTOS, E. P. 1978. Dinâmica de populações aplicada a pesca e piscicultura. São Paulo, HUCITEC, EDUSP, 129p.

SANTOS, M. C. F., A. E. T. S. FREITAS \& J. A. D. MAGALHÃES. 2003 Aspectos biológicos do camarão sete-barbas, Xiphopenaeus kroyeri (Heller, 1862) (Crustacea: Decapoda: Penaeidae) capturado ao largo do município de Ilhéus (Bahia - Brasil). Bol. Téc. Cient. CEPENE, 11(1): 73-85.

SANTOS, M. C. F., J. C. R. SILVA \& T. A. MATOS. 2007. Aspectos da biologia pesqueira do camarão sete-barbas, Xiphopenaeus kroyeri (Heller, 1862) (Decapoda, Penaeidae), em área de influência do terminal marítimo de Belmonte (Belmonte - Bahia, Brasil). Bol. Téc. Cient. CEPENE, 15(2): 69-79.

SANTOS, M. C. F., BRANCO, J. O. \& BARBIERI, E. 2013. Biologia e pesca do camarão setebarbas nos estados nordestinos brasileiros onde não há regulamentação do período de defeso. Bol. Inst. Pesca, 39(3): 217 - 235.

SANTOS, M. C. F., COELHO, P. A. \& RAMOS- 
PORTO, M. 2006. Sinopse das informações sobre a biologia e pesca do camarão setebarbas, Xiphopenaeus kroyeri (Heller, 1862) (Decapoda: Penaeidae) no Nordeste do Brasil. Bol. Téc. Cient. CEPENE, 14(1): 141178.

SANTOS, M. C. F. \& C. T. C. IVO. 2000. Pesca, biologia e dinâmica populacional do camarão sete-barbas, Xiphopenaeus kroyeri (Heller, 1862) (Crustacea: Decapoda: Penaeidae), capturado em frente ao município de Caravelas (Bahia, Brasil). Bol. Téc. Cient. CEPENE, 8(1): 131-164.

SANTOS, E. P., G. S. NEIVA \& I. SCHAEFFER. 1969. Dinâmica da população do camarão sete-barbas, Xiphopenaeus kroyeri (Heller), na Baia de Santos. Pescarias Pesq., 2(2): 4155 .

SEVERINO-RODRIGUES, E., J. B. PITA, R. GRAÇA-LOPES, J. A. P. COELHO \& A. PUZZI. 1993. Aspectos biológicos e pesqueiros do camarão sete-barbas (Xiphopenaeus kroyeri) capturado pela pesca artesanal no litoral do estado de São Paulo. Bol. Inst. Pesca, 19(1): 67-81.

TREMEL, E. 1968. Recursos camaroneiros da costa de Santa Catarina, Brasil. Resultados preliminares da pesquisa sobre o camarãosete-barbas. Doc. Téc. CARPAS, 21(1):1-10.

VALENTINI, H., F. D'INCAO, L. F. RODRIGUES, J. E. REBELO NETO \& E. RAHNA. 1991. Análise da pesca do camarão-rosa, Penaeus brasiliensis e Penaeus paulensis, nas regiões Sudeste e Sul do Brasil. Atlântica, 13(1): 143157.

VAZZOLER, A. E. A. M. 1981. Manual de métodos para estudos biológicos de populações de peixes; reprodução e crescimento. Brasília, DF, CNPq. Programa Nacional de Zoologia, 108p.

WALFORD, L. A. 1946. A new graphic method of describing the growth of animals. Biol. Bull., 90(2): 141-147. 